19 Revue d'histoire du XIXe siècle

Société d'histoire de la révolution de 1848 et des

révolutions du XIXe siècle

18 | 1999

Varia

\title{
Le mot du Président
}

Jean-Luc Mayaud

URL : http://journals.openedition.org/rh19/144

DOI : 10.4000/rh19.144

ISSN : $1777-5329$

Éditeur

La Société de 1848

Édition imprimée

Date de publication : 1 juin 1999

ISSN : 1265-1354

Référence électronique

Jean-Luc Mayaud, "Le mot du Président », Revue d'histoire du XIXe siècle [En ligne], 18| 1999, mis en ligne le 29 juin 2005, consulté le 22 septembre 2020. URL : http://journals.openedition.org/rh19/144 DOl : https://doi.org/10.4000/rh19.144

Ce document a été généré automatiquement le 22 septembre 2020.

Tous droits réservés 


\title{
Le mot du Président
}

\author{
Jean-Luc Mayaud
}

1 La Société d'histoire de la révolution de 1848 et des révolutions du XIXe siècle aura donc rempli sa mission à l'occasion du cent cinquantenaire de 1848. Elle s'est mobilisée sur plusieurs front: son propre colloque, à l'Assemblée nationale, la publication de trois numéros successifs de la Revue d'histoire du XIXe siècle et une présence systématique à la quasi totalité des manifestations scientifiques organisées en France et en Europe depuis 1997. La sortie des presses des actes du colloque, à l'automne, aux Éditions Créaphis, clôturera pour nous ces intenses mois de travail. Est-ce à dire que nous en aurons terminé avec 1848 ? bien évidemment non. Notre association travaille déjà au prochain colloque qu'elle organisera en 2001 sur le thème " Comment meurent les révolutions dans l'Europe du XIXe siècle " : un appel à communication parviendra dans le courant de la présente année à tous nos adhérents et sera publié dans le prochain numéro de notre revue. Entre temps, la Société de 1848 s'associera au colloque que préparent Michel Dobry, Alain Garrigou et Bernard Lacroix, nos collègues politistes de l'Université Paris X-Nanterre, sur " La première élection du président de la République et le suffrage universel ". Enfin, la Revue d'histoire du XIXe siècle s'efforcera de rendre compte des multiples publications d'ouvrages et d'actes de colloques annoncés, des travaux en cours sur 1848, manifestement plus nombreux - l'effet cent cinquantenaire semblant avoir déterminé ou renforcé la vocation de plusieurs jeunes thésards -, et tiendra à jour la bibliographie publiée dans les numéros 14 et 16.

2 Reste que notre société n'est pas seulement vouée à l'étude de 1848 et des révolutions : elle ambitionne d'embrasser le XIXe siècle dans sa totalité, en multipliant les approches et les réflexions. En témoignent les thèmes - passés et futurs - retenus pour la Revue d'histoire du XIXe siècle et notre participation au colloque " L'invention du XIXe siècle : le XIXe siècle par lui-même ", dont les actes viennent de paraître ${ }^{1}$. Nous nous associons avec les mêmes partenaires à la préparation d'une seconde manifestation consacrée à "L'invention du XIXe siècle : le XIXe siècle au miroir du XXe ". Surtout, comme cela avait été annoncé dans la précédente livraison de notre revue, les demi-journées d'études " re-penser le XIXe siècle " ont été mises sur pied. Une vingtaine d'entre nous, chercheurs confirmés et jeunes doctorants, se sont réunis après l'assemblée générale de la Société, le 6 mars dernier, et ont récidivé le samedi 5 juin. Le choix d'une première 
réflexion sur le temps - temps des contemporains, temps des historiens - n'est pas exclusif: ces réunions, sérieusement préparées par l'envoi de courts textes d'intervenants ${ }^{2}$, sont l'occasion de débattre des thèmes de recherche, des sources, des méthodes à l'œuvre et des outils théoriques; elles ambitionnent de traiter de l'écriture de l'histoire du XIXe siècle sous tous ses aspects et composantes. Après une période de nécessaire rodage, nous comptons bien multiplier les séances à thème, co-organisées avec les diverses équipes de recherche des universités françaises: Besançon, Lyon 2, Poitiers et Tours sont ainsi déjà candidats. Dixneuviémistes, il nous paraît indispensable de nous rencontrer périodiquement et de contribuer ensemble à une structuration de la recherche. L'entreprise est modeste, certes: elle n'est pas institutionnelle, ne repose sur aucun financement et ne développe pas de programme de recherche. Reste que la qualité des échanges, dans leur sympathique convivialité, participe au décloisonnement de la recherche sur l'histoire relative au siècle dernier.

3 Avec l'ouverture à l'automne 1999 d'un site Internet, notre Société tient les engagements pris il y a quelques mois. Ce site sera disponible à l'adresse http:// www.revues.org, complétée d'une appellation spécifique encore aujourd'hui en discussion (" rh19 " ? " 1848 " ? " histoire19 ", etc. ${ }^{3}$ ), a pour objectif de faire connaître son activité et d'augmenter sa notoriété. Mieux, nous avons pour ambition de faire de ce site un véritable outil de travail, proposant au visiteur de larges extraits des textes publiés par la Revue d'histoire du XIXe siècle. Vous y trouverez tous les résumés des articles parus, mais également l'intégralité des actualités scientifiques, des comptesrendus et des éditoriaux publiés depuis 1985. Les internautes pourront en outre consulter certains articles in extenso.

4 Le site de la Revue d'histoire du XIXe siècle s'inscrit dans une initiative plus large, à laquelle notre revue doit son adresse, facile à retenir : www.revues.org a été fondé pour héberger des publications choisies pour leur qualité scientifique. Dans cette fédération, la Revue d'histoire du XIXe siècle côtoie Ruralia, Revue de l'Association des ruralistes français et les Cahiers d'histoire, organe des universités de Lyon, Grenoble, Clermont-Ferrand, Saint-Étienne, Chambéry et Avignon. D'autres revues ont pris contact avec nous ${ }^{4}$ pour participer à cette aventure, convaincues qu'émerge enfin un authentique espace scientifique francophone vers lequel l'internaute pourra se diriger sans difficulté.

5 Cette initiative, il faut l'affirmer avec force, comble un vide. Nous espérons qu'elle vous satisfera et qu'elle permettra de faire la démonstration qu'Internet peut devenir un espace de publication scientifique de premier plan. N'hésitez pas à nous faire part de vos réactions et à nous communiquer par e-mail l'adresse électronique de collègues, français ou étrangers, qui pourraient être intéressés par ces sites. Vous pourrez recevoir une information régulière et gratuite sur la mise à jour des sites en adhérant aux listes électroniques d'information de chacun d'eux. Il suffit pour cela d'envoyer un courrier électronique à l'adresse webmaster-revues@revues.org. Écrivez le message " subscribe revues.org " pour être informé des nouveautés. N'oubliez pas d'indiquer vos coordonnées électroniques et postales dans le corps du message. Vous pouvez également vous abonner en ligne à cette liste sur le site de revues.org ${ }^{5}$.

6 L'ouverture de notre site sur Internet participe donc du développement de la Revue d'histoire du XIXe siècle. Il est évident que le support papier de notre revue n'est pas remis en cause - que ceux qui seraient réticents se rassurent - : il continuera à offrir à chacun l'intégralité des services attendus. Mais c'est un formidable impact que représente http://www.revues.org, porteur d'un considérable potentiel d'interactivité 
scientifique, et il nous faut préparer l'avenir en dépit des incertitudes du calendrier des mutations de l'édition et de la communication.

7 Je dois enfin présenter les vives excuses de la rédaction de la revue pour la bien mauvaise qualité de la précédente livraison ( $n^{\circ} 17$, Les foules au XIXe siècle). Les erreurs de pagination et de présentation, les trop nombreuses coquilles et fautes d'orthographe, inadmissibles, sont indignes de la confiance de nos lecteurs et du soutien que nous apporte le Centre national du livre. Elles nous font amèrement regretter le départ pour convenances personnelles d'Évelyne Lejeune-Resnick: professionnelle de l'édition, elle s'était bénévolement dépensée sans compter, prenant en charge la totalité du contenu rédactionnel de notre revue, nous masquant cependant les multiples difficultés de son métier. Les efforts de Carole Lécuyer-Christen et Anthony Poncier n'ont pu venir à bout de nos erreurs de coordination, de la légéreté des relectures et de l'absence de vérification finale au moment de la fabrication. Prenant acte des dysfonctionnements, déterminés à ce qu'ils ne se reproduisent plus, nous avons réorganisé la distribution des tâches au sein de la rédaction, et mis sur pied, surtout, les procédures de suivi et de correction des pages de la revue. Profitons de ce malheureux épisode pour demander à tous les auteurs de veiller à la qualité formelle des textes remis : dorénavant, la rédaction n'acceptera aucun manuscrit qui ne sera pas accompagné d'une disquette informatique. Les normes éditoriales seront précisées à la fin de chaque exemplaire de la Revue d'histoire du XIXe siècle: un " petit bréviaire du parfait correcteur " est en cours de rédaction et sera consultable sur notre site Internet ${ }^{6}$.

8 J'espère que ces changements vous donneront satisfaction et que chacun voudra bien participer au développement des activités de la Société et de la Revue d'histoire du XIXe siècle. Nous comptons sur votre fidélité et vos initiatives pour aider à la diffusion de la revue : l'aide de chacun est indispensable pour élargir notre lectorat, multiplier les abonnements et assurer publicité et rayonnement à cette revue qui est la vôtre.

\section{NOTES}

1.Alain CORBIN, Pierre GEORGEL, Stéphane GUÉGAN, Stéphane MICHAUD, Max MILNER et Nicole SAVY [dir.], L'invention du XIXe siècle. Le XIXe siècle par lui-même (littérature, histoire, société). Actes du colloque international de Paris, Musée d'Orsay, Sorbonne, 11-13 décembre 1997, Paris, Presses de la Sorbonne nouvelle/Klincksieck, 1999, 398 p. 2.Si vous souhaitez être tenu informé plus précisément, prière de me faire parvenir vos propositions et vos coordonnées (si possible avec e-mail) : Jean-Luc Mayaud, Centre Pierre Léon-ISH, 14 avenue Berthelot, 69363 Lyon cedex 07. E-mail : JeanLuc.Mayaud@ish-lyon.cnrs.fr

3.Toute suggestion est la bienvenue : même adresse que note précédente. 
4.Pour soumettre la candidature d'une revue et connaître les conditions d'entrée dans la fédération, il suffit d'envoyer un courrier électronique à l'adresse webmasterrevues@revues.org.

5.http://www.revues.org

6.Il pourra être envoyé à tous ceux qui ne disposent pas d'un accès à Internet (même adresse que note 2). 